\title{
The Application of Virtual Reality Technology in Landscape Architecture Design
}

\author{
Yanrong Bai \\ Kunming University, Kunming, China
}

Keywords: Virtual reality, Virtual technology, Landscape architecture, Garden design

\begin{abstract}
At present, the information age has come, and computer technology is constantly being updated and developed. As an important part of the computer technology field, virtual reality technology is also becoming more and more mature. Virtual reality technology combines computing technology and artificial intelligence technology. It is efficient and fast. Advantages, its powerful information processing capabilities can be applied to the design of landscape architecture, and provide continuous technical support for the application and innovation of landscape architecture design. This article first analyzes the characteristics and types of virtual reality technology, and then analyzes the application of virtual reality technology in landscape design.
\end{abstract}

\section{Introduction}

Virtual reality technology is an emerging technical discipline. It gradually emerges and develops along with the development of information technology. It integrates multiple technologies into one. It is a comprehensive discipline that changes the traditional mode of separation of man and machine. It can create a sense of designer's presence, these advantages of virtual reality technology have very important significance in the design of landscape architecture. In the design of landscape architecture, the use of virtual reality technology can enable designers to be immersive in design. It has revolutionized the traditional three-dimensional animation and various software design techniques, greatly improving the designer's work efficiency and work quality. The application of virtual reality technology in landscape design will surely bring a major innovation in design [1].

\section{Virtual Reality Technology}

VR (Virtual reality) was proposed by the founder of the American VPL company, Jaron Lanier, in the early 1980s. Its specific connotation is: comprehensive use of computer graphics system, multimedia technology, sensor technology, three-dimensional simulation technology and various display and control interface devices, through the computer-generated interactive three-dimensional environment, to provide immersive immersive technology [3]. The core essence of virtual reality is to visualize computer data in order to realize the information interaction between human subjects and virtual digital objects in the virtual environment [2].

Virtual reality technology has two properties in a narrow sense and a broad sense. In a narrow sense, virtual reality technology is a kind of experience that can make people feel in the real environment and interact with the near-real scene in the virtual environment through the virtual reality hardware device, thus creating an immersive feeling. Broadly speaking, virtual reality technology is to use various graphics software and some other simulation technologies to simulate real scenes and scenarios, including vision, hearing, touch, and what may happen in the real world [2].

\section{Features of Virtual Reality Technology}

Virtual reality technology is an emerging technical discipline. It gradually emerges and develops with the development of information technology. It integrates multiple technologies into one. It is a comprehensive discipline. It uses computer technology, simulation technology and related systems. An information technology constructed to simulate real scenes has the following characteristics: 


\subsection{Multi-Perception}

Virtual reality technology is a branch of computer technology, and it is also the innovation and development of computer technology [3]. Therefore, in addition to the general characteristics of computer technology, virtual reality technology also has multi-perceptual characteristics, specifically manifested as having the ability to listen, touch and smell and many other sensory characteristics.

\subsection{Interactivity}

The mutual characteristic of virtual reality technology is that it can realize the operability and controllability of the simulated objects, and get the feedback of the simulated environment through this operability [3]. This relationship of mutuality is the interactive characteristic.

\subsection{Autonomy}

The automatic feature of virtual reality technology refers to the fact that under the virtual state of things, it can realize the autonomous movement of things according to the laws of physical motion, and then achieve the degree of autonomous operation [2].

\subsection{Existence}

The sense of existence of virtual reality technology refers to the user's immersive experience when simulating things, thus giving the user a real sense of experience and satisfying the user's multiple needs [3]. Through this sense of presence It is difficult for users to distinguish between real environment and virtual environment.

\section{Types of Virtual Reality Technology}

\subsection{Virtual Environment Modeling Technology}

The virtual environment modeling technology is to provide users with a virtual environment similar to the real scene, and is applied to landscape garden design mainly in the use of mapping software such as 3DMAX and CAD. 3DMAX technology can realize the modeling of landscape gardens [4]. After the modeling is completed, various elements are integrated and then integrated into the three-dimensional modeling, so that a rich landscape design can be obtained; the application of CAD technology is mainly in the graphic design, and the design plan of the landscape garden is realized through the CAD software [5].

\subsection{Real-Time 3d Image Generation Technology}

One of the characteristics of the application of virtual reality technology is that the data used is very large, and this feature will bring certain difficulties to the design of landscape architecture and affect the later design effect [4]. Therefore, it is necessary to use real-time 3D image generation technology to ensure image quality.

\subsection{System Integration Technology}

The system integration technology of virtual reality technology is mainly composed of five parts, including data conversion technology, information synchronization technology, data management model, model calibration technology and recognition synthesis technology.

\section{The Application of Virtual Reality Technology in Landscape Garden Design}

The application of virtual reality technology in landscape garden design has great advantages. Using virtual reality technology, you can experience the landscape garden space from multiple angles and all directions, enrich the operation of landscape garden design, and facilitate the design of landscape gardens from details [4]. In order to facilitate the smooth implementation of landscape design, the following will specifically analyze the application of virtual reality technology in landscape design. 


\subsection{Choose the Right Virtual Reality Technology}

As mentioned earlier, there are several types of virtual reality technology. Therefore, to apply this technology to the design of landscape architecture, it is necessary to choose CAD technology and 3DMAX technology reasonably. Before the creation of the virtual environment, a three-dimensional model can be designed. The designer first has a relatively accurate understanding of the design environment. The designer can use virtual reality technology to integrate the various components of the garden such as sand, stones, plants, and building facilities [5]. The other elements are built into a simulated environment to construct a three-dimensional landscape garden model, which lays the foundation for the design in the next section.

\subsection{Establish a Three-Dimensional Model to Check the Design Effect}

When designing landscape gardens, designers must consider the impact of the surrounding environment on the garden and have a comprehensive understanding of the environment. Before designing, designers must predict and analyze the environment after completion in advance, which requires effective means to check the design effect. The traditional inspection method is sand table and renderings. This inspection method has certain subjective colors and lacks sufficient user experience. In the design of landscape architecture, it is necessary to strengthen the application of virtual reality technology and further improve the deficiencies in the traditional inspection [5]. Using virtual reality technology, a three-dimensional model can be constructed to restore the physical form of landscape architecture,shown as Fig.1. There are many components in the three-dimensional model, and these parts can be restored by computer technology, which can provide sufficient basis for the design. At present, the use of virtual reality technology to restore real objects such as stones and plants in landscape gardens is still very difficult. These objects have different shapes, and it is necessary to construct multiple faces in the three-dimensional model. Therefore, it is necessary to further research and develop virtual reality technology to improve the landscape design.

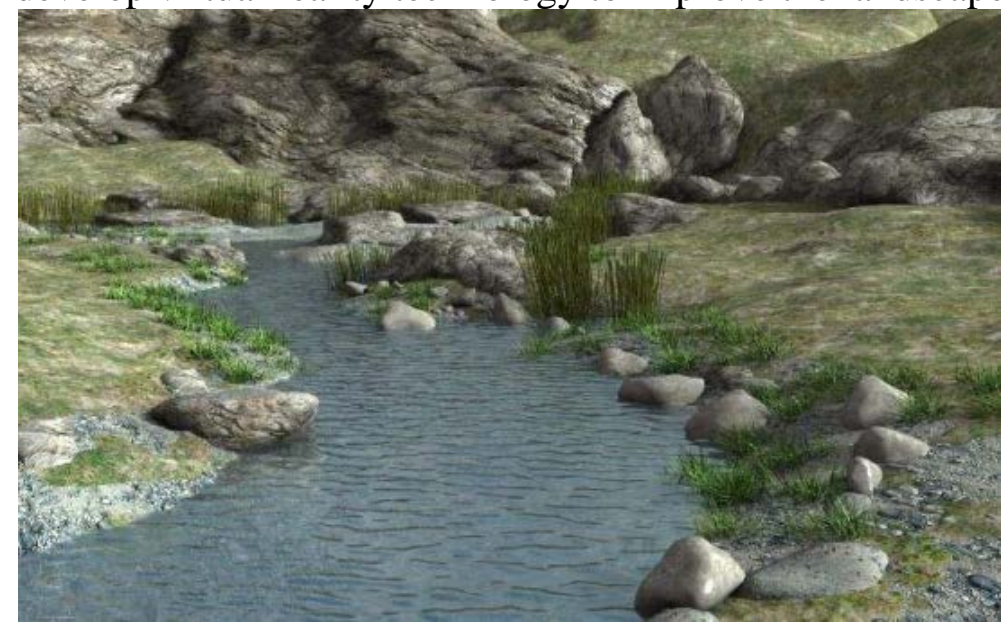

Fig.1 Virtual Reality Technology to Build 3d Model

\subsection{Virtual Scene, Enrich Browsing Content}

At present, there are various methods derived from virtual reality technology, and the common methods are VRML and 3DSMAX technology. These two technologies can improve the problems in the virtual reality technology, and the application range is very wide. VRML is mainly to create virtual and realistic scenes with the help of three-dimensional graphics language. Designers can create according to their own ideas, showing innovative ideas and brand-new design concepts. The application range of 3DSMAX technology is wider and more practical [6]. Using this technology can not only give designers more free space, but also can use this technology to control the camera to realize the navigation function in the virtual scene. The 3DSMAX technology can make browsing the content more abundant, and the above-mentioned functions can be realized by using the active control. 


\subsection{Realize an All-Round Experience and Assist in Design Decisions}

Using virtual reality technology, landscape garden design can be carried out from different angles and in different directions. Through dynamic capture and design, people can get a full range of experience. Virtual reality technology can further enrich the movement of landscape gardens, and can switch and select different sports modes. In order to bring the user a better sensory experience, parameters can be set and observations can be made from different angles to make the landscape design more reasonable [7]. It is necessary to strengthen the handling of details so that designers can observe and control the landscape garden in all directions, enrich the functions of the landscape garden, and make it more beautiful. Using virtual reality technology can also switch the season at any time, adjust the elements according to different seasons, edit the color to make it conform to the actual situation, and simulate the landscape scene to the greatest extent. Virtual reality technology can fully integrate the designer's ideas and innovative consciousness, and can also assist in decision-making and provide corresponding reference for decision-making [6]. Virtual reality technology can integrate all kinds of information and improve the landscape design plan.

\subsection{Virtual Technology Chooses the Constituent Elements of the Garden}

In the process of creating a landscape garden virtual environment, we must fully consider the constituent elements of the garden such as gravel, plants, building facilities, etc. and handle them according to the requirements. Therefore, we use virtual in the process of making three-dimensional modeling and renderings with real-world technology, it is necessary to carry out a full range of simulations from various aspects such as space, time, color, sound, etc. of the landscape garden model composition, in order to continuously enrich the content of three-dimensional modeling. In addition, various things such as gravel, plants and building facilities that are often used in garden design can be processed using VRNL technology, which can create a brand new virtual scene of garden landscape [7]. In addition, we must make full use of the camera to navigate in the three-dimensional landscape garden scene, and constantly browse the renderings to ensure the design effect of the landscape garden, as shown in Figure 2.

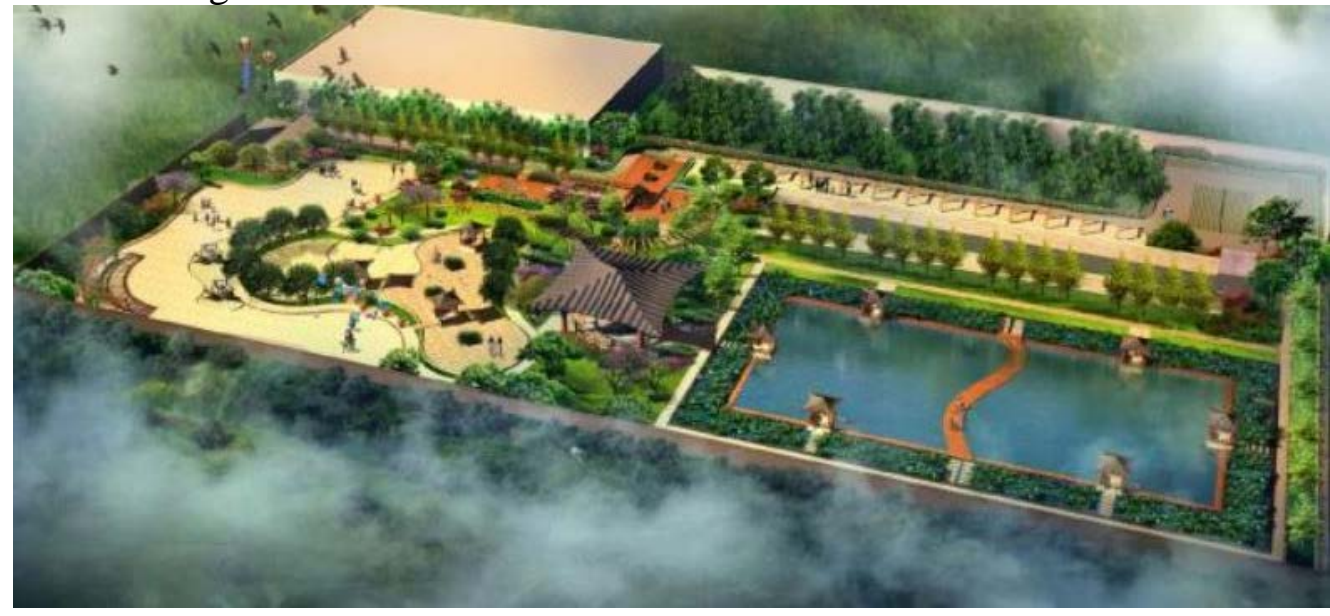

Fig.2 Virtual Technology Chooses Garden Design Elements

\section{Summary}

Virtual reality technology has a very important meaning in the design of landscape gardens, which is convenient for designers to feel immersive when designing, so that they can integrate emotional and rational factors into the design, breaking through the two-dimensional design mode of traditional design. It has realized a new design method of three-dimensional simulation space, and with the continuous development of practice and the continuous development of computer technology, virtual reality technology will play an increasingly important role in the design of landscape architecture. 


\section{References}

[1] Su, X.H. Discuss the Application of Virtual Reality Technology in landscape Design. Agriculture and Technology, vol.2, pp.112-114, 2018.

[2] Weng, N.L. Explore the Application of virtual Reality Technology in Landscape Design. Green Technology, vol.12, pp.18-21, 2017.

[3] Tang, H.L. Analysis of the Application of virtual Reality Technology in Landscape Design. Jilin Agriculture, vol.4, pp.77-80, 2018.

[4] Weng, N.T. The Application of virtual Reality Technology in Landscape Design. Agriculture and Technology, vol.5, pp.33-36, 2019.

[5] Song, X.T. Discussion on the Application of virtual Reality Technology in Landscape Design. Agriculture and Technology, vol.2, pp.44-46, 2018.

[6] Huang, J.F. Application of Application of virtual Reality Technology in Landscape Design. Engineering Design Research, vol.3, pp.32-35, 2015.

[7] Zhao, F.F. Application of Virtual Reality Technology in Landscape Architecture Design. Modern Horticulture, vol.9, pp.65-67, 2018. 